\title{
A New Approach to the Design of An Adaptive Fuzzy Sliding Mode Controller
}

\author{
Girish Vithalrao Lakhekar ${ }^{1}$ \\ ${ }^{I}$ Department of Electrical Engineering, G. H. Raisoni Institute of Engineering \& Technology, Pune, India
}

(Manuscript Received March 21 2013; Revised April 18, 2013; Accepted May 20, 2013)

\begin{abstract}
This paper presents a novel approach to the design of an adaptive fuzzy sliding mode controller for depth control of an autonomous underwater vehicle (AUV). So far, AUV's dynamics are highly nonlinear and the hydrodynamic coefficients of the vehicles are difficult to estimate, because of the variations of these coefficients with different operating conditions. These kinds of difficulties cause modeling inaccuracies of AUV's dynamics. Hence, we propose an adaptive fuzzy sliding mode control with novel fuzzy adaptation technique for regulating vertical positioning in presence of parametric uncertainty and disturbances. In this approach, two fuzzy approximator are employed in such a way that slope of the linear sliding surface is updated by first fuzzy approximator, to shape tracking error dynamics in the sliding regime, while second fuzzy approximator change the supports of the output fuzzy membership function in the defuzzification inference module of fuzzy sliding mode control (FSMC) algorithm. Simulation results shows that, the reaching time and tracking error in the approaching phase can be significantly reduced with chattering problem can also be eliminated. The effectiveness of proposed control strategy and its advantages are indicated in comparison with conventional sliding mode control FSMC technique.
\end{abstract}

Keywords: Adaptive fuzzy sliding mode control, Autonomous underwater vehicle, Depth control, Fuzzy approximator, Sliding mode control.

\section{Introduction}

In recent years, underwater robotic vehicles (URV's) have been intensively developed for their emerging applications such as scientific inspection of deep sea, long range survey, oceanographic mapping, underwater pipeline tracking, exploitation of underwater resources and so on. The characteristics of an AUV's motion depend on mode of maneuvering, forward speed, instantaneous attitude and outside appendages such as measuring instruments, tracking sonar and acoustic telemetry modem. In addition that, control issue of AUV is very challenging due to nonlinearity, time variance, unpredictable external disturbances such as the envi-

\footnotetext{
*Corresponding author. Tel.: + 91-9623611142, Fax.: +91-20-27052817, E-mail address: gv_lakhekar@rediffmail.com Copyright $\odot$ KSOE 2013.
}

ronmental force generated by the sea current fluctuation and the difficulty in accurately modeling the hydrodynamic effect. The well developed linear controllers may fail in satisfying performance requirements, especially when changes in the system and environment occur during the AUV operation. Since, it is almost impossible to manually retune the control parameters in sea. Therefore, control systems of AUV's need to have the capacities of learning and adopting to the unknown nonlinear hydrodynamic effects, parameter uncertainties, internal and external perturbations such as water current or sideslip effect. In order to deal with parametric uncertainty and highly nonlinearity in the AUV's dynamics, many researchers concentrated their interests on the applications of robust control of underwater vehicles [7],[13].

Sliding mode control has been successfully ap- 
plied for dynamic positioning and motion control of underwater vehicles, due to its robustness against modeling imprecision and external disturbances. Yoerger and Slotine [17] introduced the basic methodology of using sliding mode control for AUV application, and later Yoerger and Slotine [16] developed an adaptive sliding mode control scheme in which a nonlinear system model is used. They have investigated the effects of uncertainty of the hydrodynamic coefficients and negligence of cross coupling terms. Goheen et al. [6] have proposed multivariable self tuning controllers as an autopilot for underwater vehicles to overcome model uncertainties while performing auto positioning and station-keeping. Cristi et al. [3] proposed an adaptive sliding mode controller for AUV's based on the dominant linear model and the bounds of the nonlinear dynamic perturbations. Fossen and $\mathrm{Sa}-$ tagun [5] designed a hybrid controller combining an adaptive scheme and a sliding mode term for the motion control of a remotely operated vehicle (ROV). Healy and Lienard [9] suggested multivariable sliding mode autopilot based on state feedback for the control of decoupled model of underwater vehicles. Da Cunha et al. [4] developed an adaptive control scheme for dynamic positioning of a ROV, which is based on a sliding mode controller that only used position measurements. Lam and Ura [11] proposed nonlinear controller along with switched control law for non-cruising AUV in path following. Lee et al. [12] applied a discrete time quasi-sliding mode controller for an AUV with uncertainties of system parameters and with a long sample interval. The tracking performance enhancement for nonlinear system is carried out by tuning the parameters of sliding mode control strategy. A moving sliding surface was designed by Choi and Park [2] for fast convergence speed with rotating or shifting surface is adaptable to arbitrary initial condition. Ha [8] introduced a novel sliding mode control with fuzzy logic tuning for accelerating the reaching phase and overcome from the influence of unmodeled uncertainties, due to that robust tracking response is enhanced. Temeltas [15] employed fuzzy adapted sliding mode controller in which slope of sliding surface and discontinuous gain are tuned by fuzzy logic.

The main disadvantage of the SMC method is its dependence on system model. On the other hand, even if the system model is known then implemen- tation of SMC is possible. In addition, if all the states to be stabilized and controlled then transform the model into the canonical form. However, these conditions are not met for most AUV models. In order to overcome this problem, combine the concept of SMC and FLC. Kim Sung-Woo [10] proposed a fuzzy controller with fuzzy sliding surface for reducing tracking error and eliminating chattering problem due to that stability and robustness is improved. Song and Smith [14] introduced a sliding mode fuzzy controller that uses Pontryagin's maximum principle for time-optimal switching surface design and uses fuzzy logic to form this surface. Guo et al. [7] demonstrated the feasibility of applying a sliding mode fuzzy controller to an AUV in shallow water in order to perform line-ofsight guidance in the horizontal plane. Sebastian and Sotelo [13] designed an adaptive fuzzy sliding mode algorithm for the kinematic variables of an under-actuated underwater vehicle (UUV) maneuvered at low speed. Bessa et al. [1] proposed an adaptive fuzzy sliding mode controller based on adaptation of thin boundary layer for depth control of remotely operated underwater vehicles in presence of uncertain/disturbance effect.

This paper is organized as follows, In Section II, the system under study is stated and the basic SMC methodology is described with its stability is guaranteed by Lyapunov theory. Section III shows how fuzzy controller performed like a SMC. In Section $\mathrm{IV}$, the proposed controller is used to control vertical positioning of AUV. Conclusions are then summarized in the last section.

\section{Sliding Mode Control}

In this section, we state the design methodology of an SMC for depth control of AUV model based on a sliding surface. Consider a simplified nonlinear mathematical model of an underwater vehicle in vertical plane described equation of motion along zaxis.

$m \ddot{z}+c \dot{z}|\dot{z}|+d=u$

where, $\mathrm{u}$ is the control input (thrust force), $\mathrm{d}$ the disturbance caused by external forces, $c=0.5 \mathrm{CD}$ $\mathrm{QA}$ is the coefficient of the hydrodynamic quadratic damping and $\mathrm{m}$ represent vehicle's mass plus the hydrodynamic added mass. With respect to the dy- 
namic model, the following physically motivated assumption can be made:

\section{Assumptions 1:}

The vehicle's mass plus hydrodynamic added mass ie $m$ is time varying and unknown, but it is positive and bounded in between $0 \leq \mathrm{mmin} \leq$ $\mathrm{m}(\mathrm{t}) \leq \operatorname{mmax}$.

\section{Assumptions 2:}

The coefficient of hydrodynamic quadratic damping is time varying and unknown but it is bounded in between $\operatorname{cmin} \leq \mathrm{c}(\mathrm{t}) \leq \operatorname{cmax}$

\section{Assumptions 3:}

The disturbance effect $d(t)$ is time varying and unknown but it is bounded by a known function of $\mathrm{z}$, and $\mathrm{t}$, that is

The control problem is to synthesize a control law such that the state $\mathrm{z}$ traces the desired trajectory $\mathrm{zd}$ within the tolerance error. The error signal as $\mathrm{e}=\mathrm{z}$ $\mathrm{zd}$ and let $\mathrm{S}(\mathrm{t})$ be a sliding surface defined in the state space by the equation $S(z ; t)=0$ as

$$
\begin{aligned}
S(z ; t) & =\left(\frac{d}{d t}+\lambda\right) e(t) \\
& =\dot{e}(t)+\lambda e(t), \quad \lambda>0
\end{aligned}
$$

where, $\lambda$ is a positive constant that determines the slope of the sliding surface. The sliding mode $\mathrm{S}(\mathrm{t})=$ 0 is described by the first order equation, then

$\dot{e}(t)=-\lambda e(t)$

with the solution of above equation

$e(t)=e(0) \exp (-\lambda t)$

It is obvious from Eq. (4) that the system dynamics depend on $\lambda$. For instance, a high value of $\lambda$ is expected to move the error variables on the sliding surface faster. On other hand, if the value of $\lambda$ is chosen too high, it can cause an overshoot in the system states and instability. In general, the motion of an SMC can be divided in two modes: the reaching mode and the sliding mode. During reaching mode, the error variables are driven to the sliding surface by implementing a suitable reaching control strategy. On other hand, when the error variables are on the sliding surface, the system is said to be in the sliding mode in which the errors are driven to the origin by implementing an equivalent control strategy.

The vertical positioning of an AUV's model can be controlled by SMC under the influences of parametric uncertainty and disturbances. The control law composed of an equivalent control and a discontinuous term.

$u=c \dot{z}|\dot{z}|+d+m \lambda\left(\dot{z}_{d}-\dot{z}\right)-K \operatorname{sgn}(S / \phi)$

Where, $\mathrm{K}$ is the hitting gain, its value should be selected as a positive real number and sgn (.) is the signum function defined as

$\operatorname{sgn}(x)=\left\{\begin{array}{cc}-1 & \text { if } x<0 \\ 0 & \text { if } x=0 \\ 1 & \text { if } x>0\end{array}\right.$

It is well known that the controller in Eq. (5) suffers from high frequency switching near the sliding surface and chattering occurs due to signum function. In order to avoid chattering, a boundary layer is introduced with width. Hence, signum function can be easily replaced by a saturation function sat $(\mathrm{S} /)$ that is expressed as follows

$\operatorname{sat}(S / \phi)=\left\{\begin{array}{cc}S / \phi & \text { if }|S / \phi| \leq 1 \\ \operatorname{sgn}(S / \phi) & \text { otherwise }\end{array}\right.$

The control law is designed in such manner that, the output trajectory reaches to the sliding surface and slide on it, under that condition it will move towards equilibrium point.

Lyapunov stability analysis is the most popular approach to prove and to evaluate the stable convergence property of nonlinear controller as SMC. Here, direct lyapunov stability approach is employed to investigate the stability property of closed loop system for regulating vertical positioning of AUV model.

Theorem 1: Let the underwater vehicle be represented by in Eq. (1), then subject to Assumptions 13, the controller defined by Eq. (5), will track the 
desired trajectory asymptotically and ensures the convergence of the states to the sliding surface $S(t)$.

\section{Proof:}

We define a lyapunov function as

$V=\frac{1}{2} S^{2}$

Taking the time derivative of a lyapunov function

$$
\begin{array}{rlr}
\dot{V} & =S \dot{S} & \text { betwe } \\
& =S[\ddot{e}+\lambda \dot{e}] & \text { polic } \\
& =S\left[\left(\ddot{z}-\ddot{z}_{d}\right)+\lambda \dot{e}\right] & \text { base } \\
& =S\left[m^{-1}(-c \dot{z}|\dot{z}|-d+u)+\lambda \dot{e}\right] \\
& =S\left[m^{-1}\left(-c \dot{z}|\dot{z}|-d+\left(c \dot{z}|\dot{z}|+d+m \lambda\left(\dot{z}_{d}-\dot{z}\right)-K \operatorname{sgn}(S / \phi)\right)\right)+\lambda \dot{e}\right] \\
& =S\left[m^{-1}(-c \dot{z}|\dot{z}|-d+(c \dot{z}|\dot{z}|+d-m \lambda \dot{e}-K \operatorname{sgn}(S / \phi)))+\lambda \dot{e}\right] \\
& =S\left[-m^{-1} K \operatorname{sgn}(S / \phi)\right] \\
& =-K|S| \\
& \leq 0
\end{array}
$$

Finally, this ensures the global stability of the closed loop system and completes the proof. The SMC has robustness against uncertainty and external disturbances, Hence it is successfully employed to the dynamic positioning of AUV's. The discontinuity in the control law must be smoothed out for avoiding the undesirable chattering effects. The chattering problem is eliminated by using fuzzy sliding mode algorithm.

\section{Fuzzy Sliding Mode Control}

In this section, we follow the development established in [10] and show that a particular fuzzy controller is an extension of an SMC with a boundary layer. FSMC technique integrates both FLC and SMC. FLC techniques suffer from various problems such as (i) the design of the FLC is difficult because of theoretical analysis base is not available. (ii) the system stability is less because FLC is usually designed from an intuitive standpoint rather than a stability standpoint. (iii) The huge amount of fuzzy rules for higher order plant, increases the complexity of system analysis. To overcome the above mentioned problems, many researches are performed to design FLC's based on Lyapunov stability theory and SMC approach. It provides a simple way to achieve asymptotic stability of the system. In addition, an FSMC method includes a minimal fuzzy set of the fuzzy controller and provides systematic design procedure. In order to enhance the capability of FLC and eliminate the drawbacks, we apply the FSMC scheme which includes the advantages of FLC and SMC. The combination of FLC and SMC is based on the similarity between them that we can easily convert the SMC policy in to linguistic terms by formulating the rule base of FLC. Let the fuzzy controller in this article is constructed from the following IF -THEN rules,

\section{$\mathrm{R} 1$ : If $\mathrm{S}$ is NL then uf is $\mathrm{BB}$ \\ $\mathrm{R} 2$ : If $\mathrm{S}$ is NM then uf is $\mathrm{B}$ \\ $\mathrm{R} 3$ : If $\mathrm{S}$ is $\mathrm{ZE}$ then uf is $\mathrm{M}$ \\ $\mathrm{R} 4$ : If $\mathrm{S}$ is $\mathrm{PM}$ then uf is $\mathrm{S}$ \\ R5: If $\mathrm{S}$ is PL then uf is SS}

Equivalently,

$R^{i}$ :If Sis $F_{s}^{\prime i}$ then $u_{f}$ is $F_{u f}^{\prime \prime} \quad i=1,2,3 \ldots . .5$

where, NL is negative large, NM is negative medium, $\mathrm{ZE}$ is zero, PM is positive medium, PL is positive large, BB is bigger, $\mathrm{B}$ is big, $\mathrm{M}$ is medium, $\mathrm{S}$ is small and SS is smaller. NL, NM, ..... S, SS are labels of fuzzy sets and their corresponding membership functions are depicted in Fig. 1 and 2, respectively. Let $\mathrm{X}$ and $\mathrm{Y}$ are the input and output space of the fuzzy rules, respectively.

For any arbitrary fuzzy Fx in X, each rule Ri can determine a fuzzy set $\mathrm{Fx} * \mathrm{Ri}$ in Y. Use the sup-min compositional rule of inference and suppose $\mathrm{Fx}$ be a fuzzy singleton, then

$$
\mu_{F_{X} \circ R^{i}}\left(u_{f}\right)=\min \left[\mu_{F_{S}^{i}}(\alpha), \mu_{F_{u f}^{i}}\left(u_{f}\right)\right]
$$

the deduced membership function Fu'd of the con- 
sequence of all rules is,

$\mu_{\tilde{F}_{u_{f}}^{d}}^{d}\left(u_{f}\right)=\max \left[\mu_{\tilde{F}_{X} \circ R^{i}}\left(u_{f}\right), \ldots \ldots \mu_{\tilde{F}_{X} \circ R^{s}}\left(u_{f}\right)\right]$

where, the output variable in Eq. (11) is fuzzified output. For the defuzzifier, the centriod defuzzification method is used to find the crisp output such as

$$
\hat{u}=\frac{\int u_{f} \mu_{\tilde{F}_{u_{f}}^{d}}^{d}\left(u_{f}\right) d u_{f}}{\int \mu_{\tilde{F}_{u_{f}}^{d}}^{d}\left(u_{f}\right) d u_{f}}
$$

Then, a FSMC with variable sliding surface which satisfies the reaching condition will be designed. The reaching condition is given as

$$
S \dot{S} \leq-\eta|S| \text { for } \eta>0
$$

Fig.3 is the result of defuzzified output for a fuzzy input as sliding surface and overall control equation of fuzzy sliding mode controller

$$
\hat{u}=u_{e q}-K_{f} \operatorname{sgn}(S / \phi)
$$

The crisp control signal from extended fuzzy controller is applied to the system model for achieving stabilized response in vertical plane. Here, fuzzy control is employed as low pass filter for smoothing the control input in SMC due to that chattering problem is prevented. In this technique, minimum rules are designed to satisfy the sliding condition and also capable of adopting uncertainty in the model parameters.

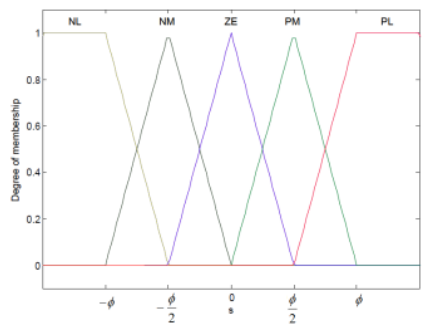

Fig.1 Membership functions for input $\mathrm{s}$

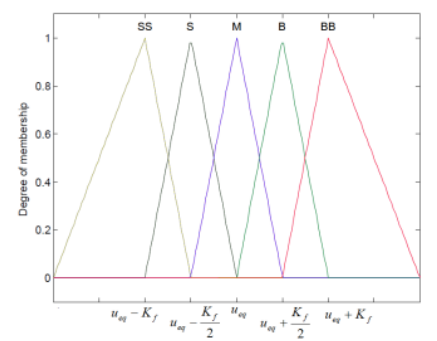

Fig.2 Membership functions for output $\mathrm{u}$
Direct Lyapunov stability approach is employed for investigating the stability of underwater vehicle in vertical plane is given as follows

Theorem 2: Consider an AUV model in vertical plane can be represented by in Eq. (1), then subject to Assumptions 1-3, the controller defined by Eq. (14), ensures the convergence of the states to the sliding surface $\mathrm{S}(\mathrm{t})$ and the desired trajectory tracking.

Let a Lyapunov function candidate $\mathrm{V}$ be defined as

$V=\frac{1}{2} S^{2}$

Taking the time derivative of a Lyapunov function

$$
\begin{aligned}
\dot{V} & =S \dot{S} \\
& =S[\ddot{e}+\lambda \dot{e}] \\
& =S\left[\left(\ddot{z}-\ddot{z}_{d}\right)+\lambda \dot{e}\right] \\
& =S\left[m^{-1}(-c \dot{z}|\dot{z}|-d+\hat{u})+\lambda \dot{e}\right] \\
& =S\left[m^{-1}(-c \dot{z}|\dot{z}|-d+f \operatorname{smc}(S, t))+\lambda \dot{e}\right] \\
& =S\left[m^{-1}\left(-c \dot{z}|\dot{z}|-d+u_{e q_{-} f u z z y}-K_{f u z z y} \operatorname{sgn}(S / \phi)\right)+\lambda \dot{e}\right] \\
& =S\left[\hat{f}+\hat{d}-K_{f u z z y}(e, \dot{e}, \lambda) \operatorname{sgn}(S / \phi)\right]
\end{aligned}
$$

This ensures the global stability of the closed-loop system and completes the proof. In this section, FLC scheme have been used as a direct controller, in which, the FLC is non-adaptive in nature. 
A FLC is called non-adaptive if all of its parameters, i.e. scaling factors, membership functions and rules are kept fixed during the operation of the controller. Here, an adaptive FLC is used to fine tunes scaling factor, varying support of input-output membership function for improving the output trajectory performance. Therefore, FLC employed as a supervisory control with the FSMC in proposed control algorithm.

\section{Adaptive Fuzzy Sliding Mode Control}

In this section, we proposed an adaptive fuzzy sliding mode control for improving output trajectory response in depth control of an AUV model. The AFSMC algorithm is developed in two stages, which employed fuzzy approximator for adaptation of input and output variables of fuzzy inference system. In first stage, slope of sliding surface is adapted by using first fuzzy approximator, due to which sliding surface continuously move such that fast tracking response is obtained. Now consider the sliding surface depicted in Fig. 4, from moving sliding surface it is clear that $\lambda$ min leads to slower error convergence and longer tracking time. On other hand, the controller with $\lambda$ max leads to faster error convergence, but tracking accuracy can be degraded. Therefore, it is obvious that there is a trade-off between error convergence time and tracking time. The movement of the sliding surface can be achieved if the value of $\lambda$ is updated according to the values of the error dynamics. Note that for the sake of the stability the positiveness of $\lambda$ must be preserved during this rotation process or movement. The rotating sliding surfaces is shown in Fig. 4, in which region for possible slopes shown by stable zone of phase plane (i.e. second and fourth quadrants). System performance is sensitive to the sliding surface slope. If large values of $\lambda$ are available then system will be more stable but tracking response may be degraded because of a longer reach-

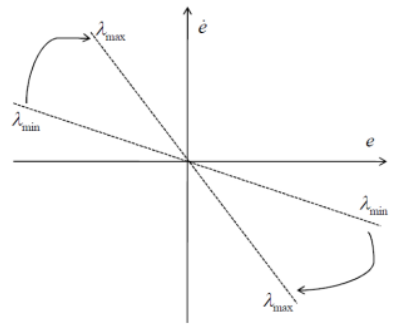

Fig.4 Rotation of sliding surface

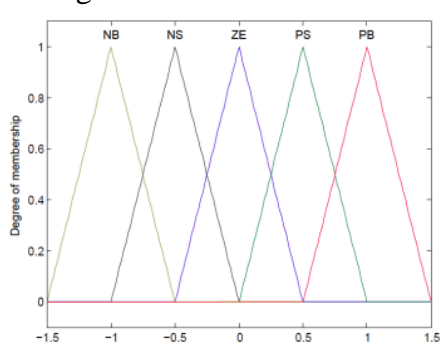

Fig.5. Membership function of input variable as error signal (e) ing time from the state to the sliding surface. Conversely, if small values of $\lambda$ are chosen then the convergence speed on the sliding surface itself will be slow, leading to longer tracking time. The slope of sliding surface is computed by fuzzy logic approximator will be represented with their input and output variables of fuzzy inference system as shown in Fig. 5 and 6.

The formulation of fuzzy rule for tuning scheme is based on concepts that a large value of $\lambda$ causes long reaching time therefore we choose small value of $\lambda$ for shorten reaching time with condition is that error is large. Input error is decomposed in to three fuzzy partitions expressed as Negative Big (NB), Negative Small (NS), Zero (ZE), Positive Small (PS) and Positive Big (PB). Output is a singleton function expressed as Small (S), Medium (M) and Large (L). The slope inference system is constructed by the idea of decreasing trajectory convergence time.

The fuzzy logic rule base is designed as follows Rule (i): If e is F i1 then $\lambda \mathrm{i}$ is $\varphi \mathrm{i}$ where, F il, $i=1,2, \ldots \mathrm{m}$. are the labels of two input fuzzy sets characterized by fuzzy membership function and $\varphi \mathrm{i}, \mathrm{i}=1,2, \ldots \mathrm{m}$ are the singleton control action. The sliding inference rules are composed as in Table I. The defuzzification of the output is accomplished by the method of centre of gravity.

$\lambda_{f u z z y}=\frac{\sum_{i=1}^{m} w_{i} \varphi_{i}}{\sum_{i=1}^{m} w_{i}}$

The crisp output variable $\lambda$ fuzzy of fuzzy logic tuning scheme is used for rotation of sliding hyperplane results in decreasing the reaching time and improving system performance.

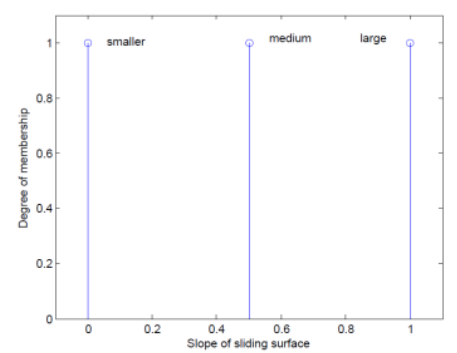

Fig.6. Membership function of output variable as slope $(\lambda)$ 
Table I Rule base for estimating sliding slope

\begin{tabular}{|l|l|l|l|l|l|}
\hline & & & & & \\
Error (e) & NB & NS & ZE & PS & PB \\
\hline Slope $(\lambda)$ & & & & & \\
\hline
\end{tabular}

Here, a single-input fuzzy adaptation is used to continuously compute the slope of the sliding surface, with the result that the sliding surface is rotated in such direction that tracking performance of the system under the control is enhanced. In the second stage, we propose a dynamic fuzzy logic tuning method for adjusting the supports of the output membership function based on the information of error dynamics. The output membership function of fuzzy inference system composed of two factor such as equivalent control ueq and hitting gain Kf, which is shown in Fig. 2. Here, a supervisory fuzzy inference system is used to adaptively tune the hitting gain in order to improve the approaching angle towards sliding surface.

The principal of operation can be easily understood from the block diagram of fuzzy inference system as shown in Fig. 7, in which dynamic tuning is used to update the support of output membership function. In path tracking application, however, the system invariance properties are observed only during the sliding phase, but in reaching phase, tracking may be hindered by disturbances or parameter variations. The straightforward way to reduce tracking error and reaching time by increasing hitting gain, which may causes chattering effect. The chattering can also be reduced by using small

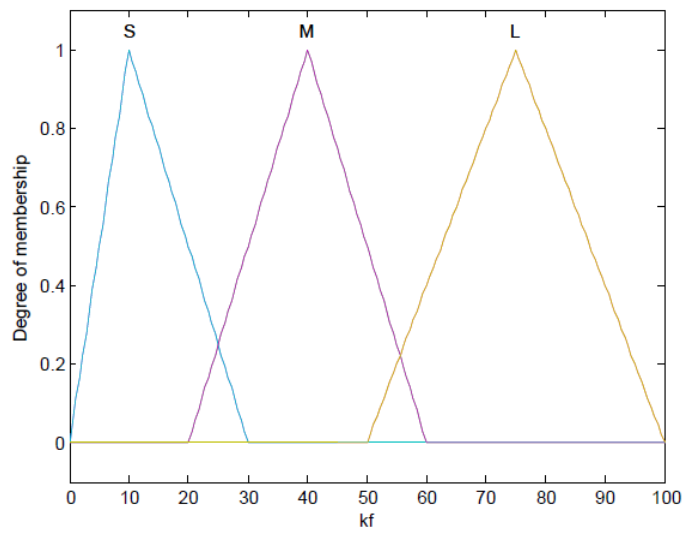

Fig. 8 Membership function of hitting gain

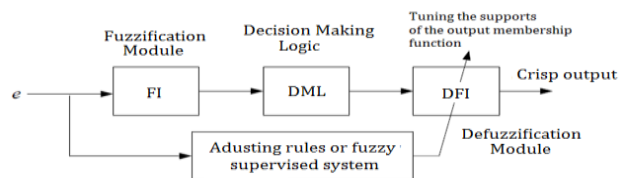

Fig. 7 Block diagram of fuzzy inference module involved in FSMC algorithm

boundary layer thickness. The selection of hitting gain value is based on minimization of tracking error and reaching time, whenever the tracking error is negative then we have to choose small gain value for desired performance of system and vice versa. The sliding hyperplane highly depend upon dynamics of error and change in error so that we have to consider error as input variable to the fuzzy logic module for updating hitting gain. The adjusting rules are designed such that, as the value of e is in large level then required control effort is bigger, due to which speed of convergence is increased. Therefore, we have to shift the support of output membership function towards the right for providing large control forces. As the value of e is near to zero then the output membership functions return back to the original type to prevent the happening of overshoots and keep the tracking accuracy. In this stage, hitting gain is adapted from the second fizzy approximator due to which support of output membership function is indirectly tuned. This output membership function is the part of fuzzy inference engine, which included in FSMC algorithm. The second fuzzy approximator plays an important role in an indirect type of adaptation, which has error as input variable and hitting gain is output variable.

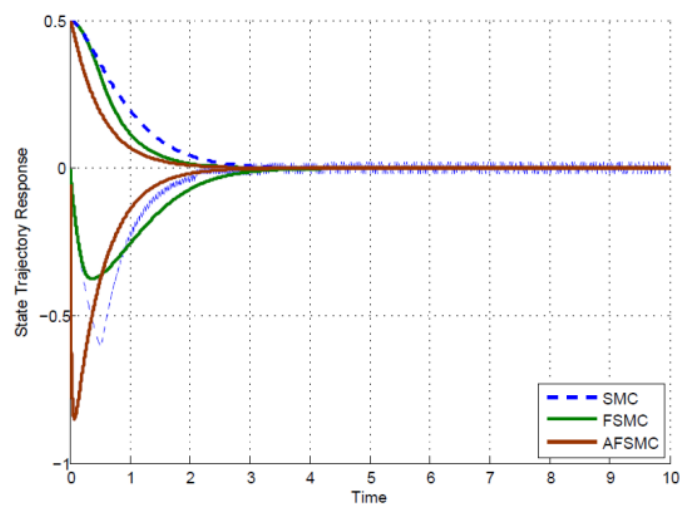

Fig. 9 State trajectory response of AUV in vertical plane 


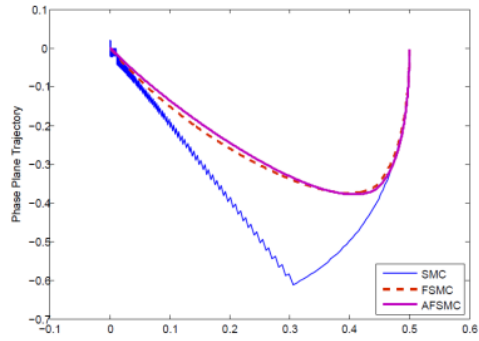

Fig. 10 Phase trajectory response of AUV in vertical plane

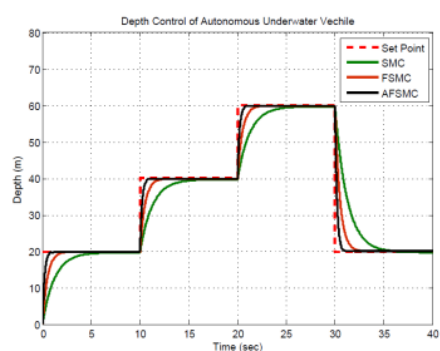

Fig. 11 Set point tracking response of Fig. 12 Response of AUV under the AUV in vertical plane

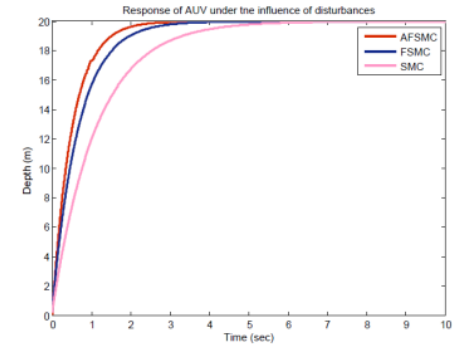

influence of disturbance

By using above consideration, the general rule is composed as, if the error signal in negative region then select small value of hitting gains and vice versa. Let $\mathrm{e}$ is the error signal as the input linguistic variable of fuzzy logic is as shown in Fig. 5 and the hitting control gain $\mathrm{Kf}$ be the output linguistic variable is as shown in Fig. 8, the associated fuzzy sets for e and $\mathrm{Kf}$ are expressed as follows:

The error signal (e) as antecedent proposition can be expressed in to five fuzzy partitions such as Negative Big (NB), Negative Small (NS), Zero (ZE), Positive Small (PS) and Positive Big (PB). The hitting control gain $\mathrm{Kf}$ as consequent proposition can be expressed in to three fuzzy partitions such as Small (S), Medium (M) and Large (L). Then, fuzzy linguistic rule base can be design as follows:

Rule 1: If e is NB then $\boldsymbol{K}_{f}$ is $\mathrm{S}$

Rule 2: If e is NS then $\boldsymbol{K}_{f}$ is $\mathrm{S}$

Rule 3: If e is ZE then $\boldsymbol{K}_{f}$ is M

Rule 4: If e is PS then $\boldsymbol{K}_{f}$ is L

Rule 5: If e is PB then $\boldsymbol{K}_{\boldsymbol{f}}$ is L

In this study, centriod defuzzification method is adopted for estimation of hitting control gain through fuzzy logic inference mechanism. Moreover, the stability of the underwater vehicle in vertical plane can be analyzed by direct lyapunov function approach, which uses AFSMC algorithm. In this investigation, each rule is applied to common lyapunov function.

Select a lyapunov function as follows

which is obviously positive definite and differentiable.

Then, its derivative can represented as

$$
\begin{aligned}
\dot{V} & =z \dot{z}+\dot{z} \ddot{z} \\
& =z \dot{z}+\dot{z}\left[m^{-1}(-c \dot{z}|\dot{z}|+\hat{u})\right] \\
& =z \dot{z}+\dot{z}\left[m^{-1}\left(-c \dot{z}|\dot{z}|+u_{e q_{-} f u z z y}-K_{f u z z y} \operatorname{sgn}(S / \phi)\right)\right] \\
& =z \dot{z}+\dot{z}\left[m^{-1}\left(-c \dot{z}|\dot{z}|-K_{f u z z y}(e, \dot{e}, \lambda)|z|\right)\right]
\end{aligned}
$$

For Rule (1), Kfuzzy $=10$ and e $=(\mathrm{z}-\mathrm{zd})=[-1.5-$ $0.5]$

$$
\dot{V}=z \dot{z}+\dot{z}\left[m^{-1}(-c \dot{z}|\dot{z}|-10 *|z|)\right] \leq 0
$$

For Rule (2), Kfuzzy $=10$ and e $=(\mathrm{z}-\mathrm{zd})=\left[\begin{array}{ll}-1 & 0\end{array}\right]$

$$
\dot{V}=z \dot{z}+\dot{z}\left[m^{-1}(-c \dot{z}|\dot{z}|-10 *|z|)\right] \leq 0
$$

For Rule (3), Kfuzzy $=40$ and e $=(\mathrm{z}-\mathrm{zd})=[-0.5$ $0.5]$

$$
\dot{V}=z \dot{z}+\dot{z}\left[m^{-1}(-c \dot{z}|\dot{z}|-40 *|z|)\right] \leq 0
$$

For Rule (4), Kfuzzy $=80$ and e $=(\mathrm{z}-\mathrm{zd})=\left[\begin{array}{ll}0 & 1\end{array}\right]$

$$
\dot{V}=z \dot{z}+\dot{z}\left[m^{-1}(-c \dot{z}|\dot{z}|-80 *|z|)\right] \leq 0
$$

For Rule (5), Kfuzzy $=80$ and $\mathrm{e}=(\mathrm{z}-\mathrm{zd})=[0.5$ $1.5]$ 


$$
\dot{V}=z \dot{z}+\dot{z}\left[m^{-1}(-c \dot{z}|\dot{z}|-80 *|z|)\right] \leq 0
$$

Hence, all of the five rules in the FLC can lead to stabilize underwater vehicle and completes the proof.

\section{Simulation Results}

In this section, some simulation results are provided to demonstrate the effectiveness and robustness of the proposed adaptive control technique. Depth control of an AUV is chosen as an example for simulation purpose, which can be represented by simplified nonlinear equation having one degree of freedom and described by Slotine [17]. Here, the main objective is to control vertical motion of underwater vehicle by using adaptive fuzzy sliding mode method. This control technique is applicable to nonlinear AUV model, because conventional linear control can't handles nonlinearity, modeling error, parametric variation and disturbances. The underwater vehicle system can be represented in the standard form

$$
\begin{aligned}
& \dot{x}_{1}=x_{2} \\
& \dot{x}_{2}=\frac{-c}{m} x_{2}^{2} \operatorname{sign}\left(x_{2}\right)+\frac{1}{m} u-\frac{1}{m} d
\end{aligned}
$$

where, $[\mathrm{x} 1, \mathrm{x} 2]=[\mathrm{z} \mathrm{dz} / \mathrm{dt}]$ is the state vector with initial condition $x(0), u$ is the control input vector and $d(t)$ is the external disturbance. The vehicle mass $\mathrm{m}$ and drag coefficient are assumed to be bounded as $1 \leq \mathrm{m} \leq 5$ and $0.5 \leq \mathrm{c} \leq 1.5$. The actual values of $\mathrm{m}, \mathrm{c}$ and $\mathrm{d}$ are used in the simulation are

$$
\begin{aligned}
& m=3+1.5 \sin (|\dot{z}| t) ; c=1.2+0.2 \sin (|\dot{z}| t) \\
& d=20+\sin (10 t)
\end{aligned}
$$

The propeller of the underwater vehicle can provide a bounded force which in turn can produce vertical motion. It is assumed here that motion remains within the operational range and the initial conditions for the states as [0 0.5$]$ and for the control is zero.For a definite class of nonlinear systems there is an appropriate robust control method called sliding mode control. This control method can be applied very well in the presence of model uncertainties, parameter fluctuations and disturbances provided that the upper bounds of their absolute values are known. The SMC is especially appropriate for the tracking control of robotic application, but the disadvantage of this method is the drastic changes of the manipulated variable. However, this can be avoided by a small modification: a boundary layer is introduced near the switching line which smoothes out the control behavior and ensures the states remaining within the layer.

Fuzzy controllers work like modified SMCs and its structure is derived from a non-linear state equation representing a large class of physical systems. Furthermore, the following aspects are discussed: stability conditions, scaling (normalization) of the state vector and choice of the switching line. By the choice of an additional boundary layer in the phase plane then fuzzy control is modified so that drastic changes of the manipulated variable can be avoided especially at the boundary of the normalized phase plane. High similarity becomes apparent when comparing the SMC with boundary layer to an fuzzy control, whose rules have been derived from the phase plane. An analysis of the two working principles leads to the conclusion that Fuzzy logic control, in the above mentioned sense, is an extension of sliding mode with boundary layer. Meaning of above aspect is that, extended fuzzy control prevent chattering problem when state of system moves on sliding surface. The extended fuzzy control is also called as modification of conventional SMC or fuzzy sliding mode control. Here, the main focus of this paper is to design adaptive strategy composed of two stages, in which fuzzy adaptation of input and output variable of fuzzy inference engine in FSMC algorithm are carried out by fuzzy approximator. First fuzzy approximator is used to estimate slope of sliding surface as per the status of state dynamics which described in terms of error signal value. While, second fuzzy approximator is employed to tune support of output membership function, which mainly composed of two factors such as equivalent control and hitting gain. Tuning of support is provided through fuzzy adaptation of hitting control gain. Due to that adaptation scheme, output tracking performance is enhanced during the parameter uncertainty and disturbance occurrences.

In order to evaluate control system performance by using proposed control algorithm is tested on 
simulated model of AUV. The performance of developed advance control method has been compared with SMC and FSMC. For comparative analysis, state trajectory and the phase portrait obtained with SMC, FSMC and AFSMC are presented in Fig. 9 \& Fig. 10 respectively. The chattering problem was not observed in both FSMC and AFSMC method. Because, in the design of fuzzy control of selecting boundary layer in the phase plane. The improved tracking performance of AFSMC with fuzzy adaptation of $\lambda$ and $\mathrm{Kf}$, compared with conventional SMC and FSMC as shown in Fig. 11. In the simulation, disturbance is added in the AUV model then proposed control technique and SMC allows the underwater vehicle to track the desired trajectory as presented in Fig. 12. The given nonlinear control strategies such as SMC, FSMC and AFSMC are capable of handling disturbances and nonlinearity. From simulation results, it is clear that proposed AFSMC provide desired tracking response with smooth control signal and minimum reaching time during model uncertainties and disturbances in operating condition.

\section{Concluding Remarks}

This study has demonstrated the effectiveness and robustness of an adaptive fuzzy sliding mode controller for regulating vertical motion of an underwater vehicle. The fuzzy logic approximators were used for enhance the tracking performance of AUV by adapting an input and output parameter as slope of sliding surface and hitting control gain of fuzzy inference engine. Due to fuzzy tuner, sliding surface continuously moving for significantly reduces reaching time and substantially enhances the system performance which is insensitive to the parameter variations and disturbance effects. The hitting control gain is adapted for tuning the support of output membership function, due to which an optimum approaching angle towards sliding surface was determined. The stability and convergence properties of the closed-loop systems were analytically proved using Lyapunov stability theory. Through numerical simulations, the improved performance over the conventional SMC and FSMC was demonstrated.

\section{References}

[1] Bessa W. M., Dutra M. S., Kreuzer E., "Depth control of remotely operated underwater vehicles using an adaptive fuzzy sliding mode con- troller", Journal of Robotics and Autonomous Systems, Vol. 56, pp. 670-677, 2008.

[2] Choi, S. B., Park, D. W., \& Jayasuriya, S., “A time-varying sliding surface for fast and tracking control of second-order dynamic systems", Automatica, Vol.30, pp. 899-904, 1994.

[3] Cristi R., Papoulias F. A. and Healey A. J., "Adaptive sliding mode control of autonomous underwater vehicles in the dive plane", IEEE J. Oceanic Engg., Vol.15, pp. 152-160, 1990.

[4] Da Cunha, J.P.V.S., Costa R. R., Hsu L., “Design of high performance variable structure control of ROV's", IEEE J. Oceanic Engg., Vol.20, No.1, pp. 42-55, 1995.

[5] Fossen, T.I., Sagatun, S., "Adaptive control of nonlinear systems: A case study of underwater robotic systems" Journal of Robotic Systems, Vol.8, pp 393-412, 1991.

[6] Goheen K. R., Jefferys E. R., "Multivariable self tuning autopilots for autonomous underwater vehicles", IEEE J. Oceanic Engg., Vol.15, No.3, pp. 144-151, 1990.

[7] Guo J., Chiu F. C., Huang C. C., "Design of a sliding mode fuzzy controller for the guidance and control of an autonomous underwater vehicle", Journal of ocean engineering, Vol. 30, pp. 2137-2155, 2003.

[8] Ha, Q. P., "Robust sliding mode controller with fuzzy tuning", IEE Electron. lett., vol. 32, pp.1626-1628, 1996.

[9] Healey A. J., Lienard D., "Multivariable sliding mode control for autonomous diving and steering of unmanned underwater vehicles", IEEE J. Oceanic Engg., Vol.18, No.3, pp. 327339,1993.

[10]Kim S. W., Lee J. J., "Design of a fuzzy controller with fuzzy sliding surface", Journal of fuzzy sets and systems, Vol. 75, pp 359-367, 1995.

[11]Lam W. C., Ura T., "Nonlinear controller with switched control law for tracking control of noncruising AUV', Int. proc. of IEEE AUV'96, California, 1996.

[12]Lee P.M., Hong S.W., Lim Y.K., Lee C.M., Jeon B.H., and Park J.W., "Discrete-time quasisliding mode control of an autonomous underwater vehicle" IEEE Journal of Ocean Engineering, Vol. 24, No.3, pp. 88-395, 1999.

[13]Sebastian E., Sotelo M. A., “Adaptive fuzzy sliding mode controller for the kinematic varia- 
bles of an underwater vehicle", J. Intelligent Robot system, Vol. 49, pp. 189-215, 2007.

[14]Song F., Smith S. M., "Design of sliding mode fuzzy controllers for an autonomous underwater vehicle without system model", MTS/IEEE Ocean Conference,Vol. 2, pp. 835-840, 2000.

[15]Temeltas H., "A fuzzy adaptation technique for sliding mode controllers", IEEE International symposium, Vol. 1, pp 110-115, July 1998.
[16]Yoerger D. and Slotine J., "Adaptive sliding control of an experimental underwater vehicle", Int. proc. IEEE Conference on Robotics and Automation, Sacramento C. A., pp 2746-2751, 1991.

[17]Yoerger D. and Slotine J., "Robust trajectory control of underwater vehicles", IEEE J. Oceanic Engg., Vol.10, No.4, pp. 462-470, 1985. 